\begin{tabular}{|l|l|}
\hline POS0359 & MOLECULAR PROFILING OF RADIOGRAPHIC AXIAL \\
& SPONDYLOARTHRITIS PATIENTS REVEALS AN \\
& ASSOCIATION BETWEEN INNATE AND ADAPTIVE \\
& CELL POPULATIONS AND THERAPEUTIC RESPONSE \\
& TO ADALIMUMAB
\end{tabular}

D. Sobral ${ }^{1}$, A. F. Fernandes ${ }^{2}$, A. Mashayekhi Sardoo ${ }^{3}$, M. Bernardes ${ }^{4}$, P. Pinto ${ }^{5}$, H. Santos ${ }^{6}$, J. L. Gomes ${ }^{7}$, J. Tavares-Costa ${ }^{8}$, J. Silva ${ }^{9}$, J. Madruga Dias ${ }^{10}$, A. Bernardo ${ }^{5}$, J. Gaillard ${ }^{11}$, J. Armengaud ${ }^{11}$, V. Benes ${ }^{12}$, R. Pinheiro Torres ${ }^{7,13}$, L. Domingues $^{13}$, S. Maia ${ }^{13}$, J. Branco ${ }^{7,13,14}$, A. V. Coelho ${ }^{2}$, F. Pimentel dos Santos ${ }^{7,13,14} .^{1}$ Faculdade de Ciências e Tecnologia, Ciências e Tecnologia, Caparica, Portugal; ${ }^{2}$ ITQB NOVA, Instituto de Tecnologia Química e Biológica, Oeiras, Portugal; ${ }^{3}$ University of Trás-os-Montes and Alto Douro, Ciências e Tecnologia, Vila Real, Portugal; ${ }^{4}$ São João Universitary Hospital Center, Rheumatology, Porto, Portugal; ${ }^{5}$ Centro Hospitalar Vila Nova de Gaia / Espinho Unit 1, Rheumatology, Vila Nova de Gaia, Portugal; ${ }^{6}$ Rheumatology Institute (IPR), Rheumatology, Lisboa, Portugal; ${ }^{7}$ Hospital Egas Moniz, Rheumatology, Lisboa, Portugal; ${ }^{8}$ Unidade Local de Saúde do Alto Minho, Rheumatology, Ponte de Lima, Portugal; ${ }^{9}$ Centro Hospitalar e Universitário de Coimbra, Rheumatology, Coimbra, Portugal; ${ }^{10}$ Centro Hospital Médio Tejo, Rheumatology, Tomar, Portugal; ${ }^{11}$ Service de Pharmacologie et Immunoanalyse (SPI), Laboratoire Innovations technologiques pour la Détection et le Diagnostic, Avignon, France; ${ }^{12}$ Genomics Core Facility, Genomics Core Facility, Heidelberg, Germany: ${ }^{13} \mathrm{CEDOC}$, Rheumatology, Lisboa, Portugal; ${ }^{14}$ Nova Medical School, Rheumatology, Lisboa, Portugal

Background: The response to treatment in spondylarthropaties is heterogeneous, due to factors yet to be better described. For that reason, it is important to find tools that might help clinicians to decide what is the best available therapeutic option for each patient.

Objectives: The goal of this study is to use comprehensive molecular profiling to characterize clinical response to therapy in a real-world setting. Specifically, to identify molecular biomarkers differentiating good responders and non-responders to TNF inhibitors (TNFi) treatment, using adalimumab, in radiographic axial spondyloarthritis I ankylosing spondylitis ( $\mathrm{r}$-axSpAIAS) patients context.

Methods: Whole-blood mRNA and plasma proteins were measured in a cohort of biologic naïve $r$-axSpAIAS patients $(n=35)$ from the Bioefficacy study (Biomarkers identification of anti-TNF alpha agent efficacy in AS patients using RNA sequencing and mass spectrometry), pre and post (14 weeks) TNFi treatment using adalimumab. Response to treatment was categorized according to ASAS20. Results of differential expression analysis were used to identify the most enriched pathways and in predictive models to distinguish responses to TNFi.

Results: A treatment-related signature, independent of the type of response, suggests a reduction in inflammatory disease activity. We found genes and proteins robustly differentially expressed between baseline and week 14 in responders, including the GWAS AS-associated genes TNFRSF1A, FCGR2A, TYK2, TBKBP1, ILIR1, IL6R, ICOSLG, IL7R, HHAT and LTBR. Moreover, CRP and HP proteins showed strong and early decrease in the plasma of AS patients, while a cluster of apolipoproteins (APO1, APO2, APO3) showed an increased expression at week 14. Good responders to TNFi treatment tend to have higher expression of innate immunity genes at baseline, and lower expression of markers associated with adaptive immunity, particularly B-cells. A logistic regression model incorporating ASDAS-CRP, gender and Gene $x$, the top differentially expressed gene at baseline between responders and non-responders, enabled an accurate prediction of response to adalimumab in our cohort (AUC=0.97).

Conclusion: Differences in disease activity and/or innate/adaptive immune cell type composition at baseline may be a major contributor to response to adalimumab in r-axSpAIAS. Alternatively, a model including clinical and gene expression variables could be considered, particularly in patients with mild disease activity. Disclosure of Interests: None declared DOI: 10.1136/annrheumdis-2021-eular.3683

\section{POS0360 \\ COMPLEX LANDSCAPE OF BIRC5/SURVIVIN GENOME BINDING IN HUMAN CD4+ T CELLS}

N. Oparina ${ }^{1}$, M. Erlandsson ${ }^{1}$, V. Chandrasekaran ${ }^{1}$, K. M. Andersson ${ }^{2}$ A. Damdimopoulos ${ }^{3}$, S. Töyrä Silfverswärd ${ }^{4}$, G. Katona ${ }^{5}$, M. I. Bokarewa ${ }^{1}$. ${ }^{1}$ University of Gothenburg, Department of Rheumatology and Inflammation Research, Institute of Medicine, Gothenburg, Sweden; ${ }^{1}$ University of Gothenburg, Department of Rheumatology and Inflammation Research, Institute of Medicine, Gothenburg, Sweden; ${ }^{3}$ Karolinska Institutet, Department of Bioscience and Nutrition, Huddinge, Sweden; ${ }^{4}$ Sahlgrenska University Hospital, Rheumatology Clinic, Gothenburg, Sweden; ${ }^{5}$ University of Gothenburg, Department of Chemistry and Molecular Biology, Gothenburg, Sweden

Background: Survivin, coded by BIRC5 gene, is a multitasking protein essential for cell renewal and homeostasis. In autoimmune conditions as rheumatoid and psoriasis arthritis, survivin was associated with inflammation severity and joint damage. Importantly, inhibition of survivin alleviated experimental arthritis in mice. We have recently shown survivin to be essential for $\mathrm{T}$ cell differentiation and microRNA processing. The known anti-apoptotic and proliferation facilitating functions of survivin does not explain the nuclear localization of survivin in interphase.
Objectives: We aimed to uncover nuclear functions of BIRC5/survivin in CD4 cell of RA patients and healthy.

Methods: CD4 T cells were isolated from the peripheral blood using positive selection on magnetic beads (EasySep) and activated for $48 \mathrm{~h}$ with ConA+LPS. Chromatin immunoprecipitation (ChIP) with polyclonal anti-survivin antibodies was done in four independent samples of healthy donors $(n=5)$, healthy smokers $(n=3)$, rheumatoid arthritis $(n=3)$ and breast cancer $(n=2)$. Pooled libraries were constructed for each group and ChIPseq was carried out (Illumina). For comparative RNAseq analysis, activated CD4 T cells were incubated with or without survivin inhibitor (YM155) for 24h. State-of-theart bioinformatics pipelines were applied for NGS data and the survivin-binding peaks were used for comparison with genes, chromatin state annotation and functional geneand regulatory regions-based functional analysis. Co-localization of peaks in the whole genome and in vicinity of the differentially expressed genes (DEG) was done using ReMap integrated ChIPseq datasets for all human cells and tissues.

Results: We identified 13 thousands non-overlapping survivin ChIP-peaks ( $>3000$ peaks were present in at least 3 samples). Survivin-bound regions were enriched near the genes and promoters $(\mathrm{p}=\mathrm{e}-30$ and $\mathrm{p}=\mathrm{e}-8)$, which implied that survivin role in transcription could be mediated by known transcription factors. Thus, we analyzed survivin peaks vs binding regions of 1135 transcription regulators (TR) available in ReMap. Potential partner proteins of survivin were selected based on the enrichment of the overlapping peaks in the whole genome and in CD4-active regulatory areas. Both, strict overlaps and location within 10 and 100kb survivin peak vicinity were analyzed. This approach allowed us to select $>150$ TRs enriched in all tests. The enriched TRs were involved in immunity and RA-relevant pathways including cytokine response and production, JAK-STAT signaling, etc. Among the TRs co-localized with survivin were CHD8, MAX, EP300, BRD2, CTCF and RAD21, all responsible for chromatin architecture. Several TRs were massively enriched in the vicinity of DEGs after survivin depletion including MAX, AR, CTCF, MYC and IRF1. Search for TR binding motifs in survivin peaks supported over-representation of binding sites for IRFs $(p=e-5)$ and several proteins of the bZIP-family $(p=e-5)$.

Conclusion: Analysis of the survivin bound DNA in CD4 cells demonstrated the nonrandom distribution with specific enrichment within the regulatory elements of the genes and co-localizeation with protein partners to regulate their transcription.

Disclosure of Interests: None declared

DOI: 10.1136/annrheumdis-2021-eular.3708

\begin{tabular}{|l|l}
\hline POS0361 & DNA METHYLATION SIGNATURES CHARACTERIZE \\
PSORIASIS AND PSORIATIC ARTHRITIS IN \\
MONOZYGOTIC TWINS DISCORDANT FOR THE DISEASE
\end{tabular}

M. Vecellio ${ }^{1,2}$, A. Ceribelli ${ }^{2}$, E. Paraboschi ${ }^{3}$, N. Isailovic ${ }^{2}$, F. Motta $^{2}$, M. De Santis $^{2}$, R. Asselta ${ }^{3}$, S. Duga ${ }^{3}$, C. Selmi ${ }^{2}{ }^{1}$ University of Oxford, NDORMS, Oxford, United Kingdom; ${ }^{2}$ Humanitas Research Hospital, Rheumatology and Clinical Immunology, Rozzano, Italy; ${ }^{3}$ Humanitas University, Department of Biomedical Sciences, Pieve Emanuele, Italy

Background: Psoriatic disease is a chronic inflammatory disorder spanning from skin disease (psoriasis) to psoriatic arthritis (PsA). The genetic background is insufficient to explain disease onset as illustrated by not very informative Genome Wide Association Studies and monozygotic (MZ) twin studies recently performed. It is strongly assumed that epigenetics may contribute to disease susceptibility modulating gene expression. DNA methylation has been found involved in several autoimmune inflammatory rheumatic diseases. Here we have analysed the DNA methylation profile of a selected cohort of MZ twins discordant for psoriasis/PsA.

Objectives: To identify the methylome associated with psoriasis and PsA in the peripheral blood of $\mathrm{MZ}$ twins discordant for these conditions.

Methods: Peripheral blood from 7 couples of MZ twins discordant for psoriatic disease was collected and DNA extracted for a genome-wide evaluation of the DNA methylation profile, with the Infinium MethylationEPIC BeadChip. Minfi and the packages of the Bioconductor were used to analyse the data obtained. Quality control and exclusion criteria were applied to the raw data having a final number of 762.451 probes, which accounts for $88 \%$ of the total.

Results: The approach first identified 2564 differentially methylated positions (DMPs; ${ }^{*} p<0.005$ ) with 19 genes potentially affected (with at least two DMPs within $1 \mathrm{~kb}$ of distance), including SMAD3 and SMARCA4/BRG1 involved in the Interferon and TGF $\beta$ pathways. Gene Ontology (GO) analysis of DMP-associated genes showed a significative enrichment $\left({ }^{*} \mathrm{p}<0.005\right)$ in transcription factor binding, transcription corepressor and transcription coactivator activity, SMAD binding and histone -lysine- $\mathrm{N}$-methyltransferase activity. To further validate the results, 5'-methylcytosine immunoprecipitation (MedIP) followed by Real Time PCR was performed to assess the methylation level of SMAD3 and SMARCA4/BRG1 promoters in the same cohort of $M Z$ twins. We found significantly DNA methylation enrichment in SMARCA4/BRG1 promoter in psoriatic disease twins $(p<0.05)$ SMAD3 and SMARCA4/BRG1 mRNA expression was also assessed to evaluate any inverse correlation with promoter methylation level, on the MZ cohort used for the EPIC array $(n=4)$ and on a cohort of PsA/Ps patients $(n=8)$ and appropriate healthy controls $(n=3)$. Reduced mRNA expression $(p<0.05)$ was demonstrated for SMARCA4/BRG1 $(n=4)$. Conversely, no changes were found for SMAD3.

Conclusion: We report the first DNA methylation approach in MZ twins discordant for psoriatic disease. We believe that the observed changes in SMAD3 
and SMARCA/BRG1 genes may suggest an epigenetic imbalance of chromatin remodelling factors involved in inflammation pathways with a potential role in PsA/psoriasis immunopathogenesis.

Disclosure of Interests: None declared

DOI: 10.1136/annrheumdis-2021-eular.3948

\section{POS0362 $1 N V E S T I G A T I N G$ THE ANKYLOSING SPONDYLITIS- ASSOCIATED REGULATORY SNPS AT THE RUNX3 LOCUS WITH A FUNCTIONAL GENOMICS APPROACH}

M. Vecellio ${ }^{1}$, P. B. Wordsworth ${ }^{1}$, C. J. Cohen ${ }^{1} .{ }^{1}$ University of Oxford, NDORMS, Oxford, United Kingdom

Background: Of the $>100$ genetic associations with ankylosing spondylitis (AS) RUNX3 transcription factor (TF) involved in diverse immunological processes, is robustly $\left(10^{-15}\right)$ associated. ${ }^{1}$ The biggest challenge is to understand the mechanism behind this association. We demonstrated the association between AS and the single nucleotide polymorphism (SNP) rs4648889 located in a $2 \mathrm{~kb}$ regulatory locus upstream of the RUNX3 promoter can be explained by allele-specific effects on TF recruitment that alter gene expression, specifically in CD8+ T-cells. ${ }^{2}$ We recently dissected the full plethora of TFs affected by rs 4648889 AS-associated allele showing the NuRD complex and IRF5 differentially bound and having a crucial role in CD8+ T-cells function ${ }^{3}$. Nevertheless, other post-GWAS approaches are needed to elucidate the function of RUNX3 AS-associated SNPs (i.e. the lead SNP rs6600247). Objectives: The purpose of this work is to define the functional effect of rs6622047 in CD8+ T-cells and monocytes. In specific, we expect to define the impact of AS-associated allele to TF binding and to evaluate chromosome looping between rs6600247 and the RUNX3 promoter.

Methods: The epigenetic landscape of SNP rs6600247 was defined using Roadmap database. In vitro functional studies were performed to characterize the effects of this SNP on TFs binding. Chromosome conformation capture (3C) provided critical functional evidence for looping among AS-associated SNPs and the RUNX3 promoter. Results: (1) In silico data revealed a c-MYC ChIP-seq peak in GM12878 lymphoblastoid cells overlapping rs6600247; (2) Mobility shift assays (EMSAs) and WB-EMSAs showed reduced DNA/protein binding in the presence of the AS-risk allele in CD14+ monocytes. C-MYC binding-site is disrupted and binding abolished in the presence of the AS-risk allele; (3) $3 \mathrm{C}$ experiments indicate low interaction frequency between SNP rs6600247 and RUNX3 promoter.

Conclusion: The enhancer upstream the RUNX3 gene has a plausible functional role in AS, probably by regulating gene transcription and DNA looping. These observations are critically important in defining dysregulated pathways and potential therapeutic drug targets.

REFERENCES:

[1] IGAS et al. Nat Genet. 2013 Jul;45(7):730-8.

[2] Vecellio M. et al, Ann Rheum Dis. 2016 Aug;75(8):1534-40.

[3] Vecellio et al Arthritis Rheumatol 2020. doi: 10.1002/art.41628.

Acknowledgements: We thank Professor Julian Knight for his constructive advices and comments to the present work.

Disclosure of Interests: None declared

DOI: 10.1136/annrheumdis-2021-eular.4098

\section{Adaptive immunity ( $T$ cells and $B$ cells) in rheumatic diseases}

\section{POS0363 IDENTIFICATION OF MOLECULAR PHENOTYPES AND IMMUNE CELL INFILTRATION IN PSORIATIC ARTHRITIS PATIENTS' SKIN TISSUES BY INTEGRATED BIOINFORMATICS ANALYSIS}

T. Cheng ${ }^{1,2,3}$, S. X. Zhang ${ }^{1,2,3}$, J. Qiao ${ }^{1,2,3}$, R. Zhao ${ }^{1,2,3}$, S. Song ${ }^{1,2,3}$, Y. Zhang ${ }^{1,2,3}$, P. Zhao ${ }^{4}$, G. Y. Liu', P. F. He ${ }^{5}$, X. Li ${ }^{1,2,3} .{ }^{1}$ The Second Hospital of Shanxi Medical University, Department of Rheumatology, Taiyuan, China; ${ }^{2}$ Shanxi Li Xiaofeng Medical Groups, Department of Rheumatology, Taiyuan, China; ${ }^{3}$ Ministry of Education, Key laboratory of Cellular Physiology at Shanxi Medical University, Taiyuan, China; ${ }^{4}$ Shanxi Provincial People's Hospital, Department of Rheumatology, Taiyuan, China; ${ }^{5}$ Shanxi Medical University, Medical Data Sciences, Taiyuan, China

Background: Psoriatic arthritis (PsA) is an inflammatory musculoskeletal disease associated with cutaneous psoriasis ${ }^{1}$. Heterogeneity of clinical manifestation often makes differential diagnosis difficult ${ }^{2}$. Thus, the underlying molecular pathogenesis of PsA need to be further studied to diagnose early and ensure optimal management of arthritis and key comorbidities.

Objectives: This research was conducted to identify molecular phenotypes and immune infiltration in the skin tissues of psoriatic arthritis patients according to bioinformatics analysis.

Methods: The mRNA expression profiles of GSE13355 (116 samples), GSE14905 (56 samples) and GSE30999 (162 samples) were obtained from the publicly GEO databases. Non-negative matrix factorization (NMF), functional enrichment and cibersort algorithm were applied to illustrate the conditions of PsA patients' skin tissues for classification after screening the differentially expressed genes (DEGs) between lesion biopsy and non-lesion biopsy.

Results: Two subsets (Sub1 and Sub2) were identified and validated by NMF typing of 612 detected DEGs (Figure 1a). A total of 54 signature genes (18 in Sub1 and 36 in Sub2) were obtained (Figure 1b). GO and KEGG enrichment analysis showed the signature genes in Sub1 were mainly involved in proliferation and differentiation of immune cells, whereas genes in Sub2 were related to humoral immune response mediated by antimicrobial peptide (Figure 1c.1d) Further, immune cell infiltration results revealed Sub2 had higher levels of resting NK cells $(P<0.001)$, macrophages $\mathrm{M} 1(P<0.001)$, resting mast cells $(P<0.001)$ and regulatory T cells $(P<0.001)$ but lower concentrations of activated $\mathrm{CD} 4^{+}$memory T cells $(P<0.001)$, activated NK cells $(P<0.05)$, activated dendritric cells $(P<0.001)$ eosinophils $(P<0.05)$ and neutrophil $(P<0.001)$ (Figure $1 \mathrm{e})$

Conclusion: The pathogenesis of psoriatic arthritis is related to both cellular immunity and humoral immunity. It is indispensable to adjust the treatment strategies according to patient's immune status.

\section{REFERENCES:}

[1] Ritchlin CT, Colbert RA, Gladman DD. Psoriatic Arthritis. The New England journal of medicine 2017;376(10):957-70. doi: 10.1056/NEJMra1505557 [published Online First: 2017/03/09].

[2]Veale DJ, Fearon U. The pathogenesis of psoriatic arthritis. Lancet (London, England) 2018;391(10136):2273-84. doi: 10.1016/s0140-6736(18)30830-4 [published Online First: 2018/06/13]

a

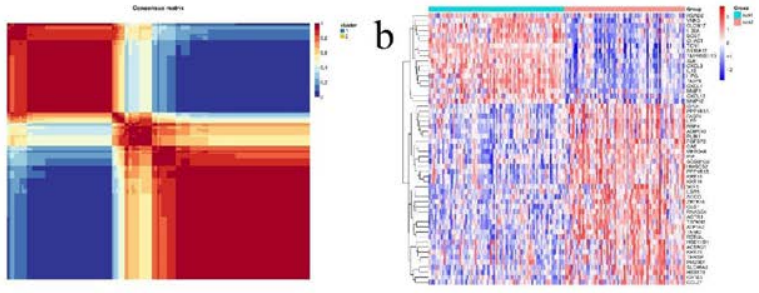

C

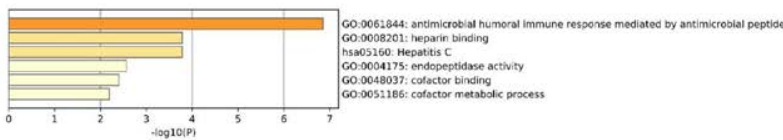

$\mathrm{d}$
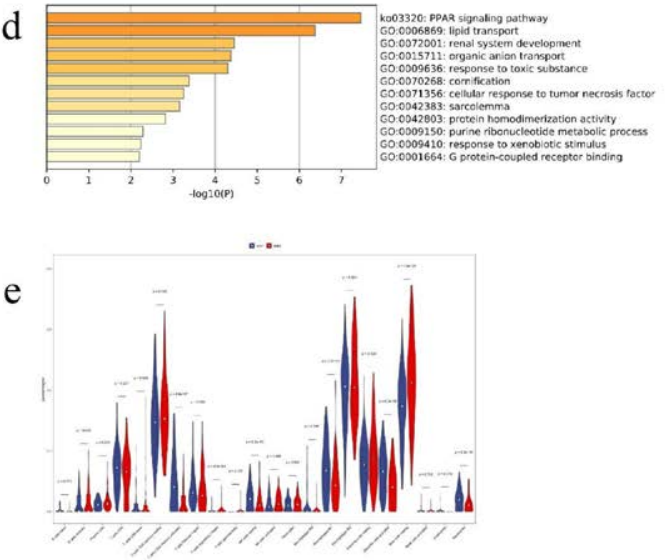

Figure (a) NMF consensus clustering of 334 PsA patients based on 612 DEGs. (b) Heat map of differentially expressed genes: Each small square represents a gene; the color indicates the expression of the gene. Each column indicates the expression level of genes in a sample; the right side is the gene name. (c.d) Visualization GO enrichment analysis and KEGG pathway enrichment. (e) Violin plot show the fraction of 22 immune cells subpopulations in two subtypes based on CIBERSORT algorithm.

Acknowledgements: This project was supported by National Science Foundation of China (82001740), Open Fund from the Key Laboratory of Cellular Physiology (Shanxi Medical University) (KLCP2019) and Innovation Plan for Postgraduate Education in Shanxi Province (2020BY078).

Disclosure of Interests: None declared

DOI: 10.1136/annrheumdis-2021-eular.2171 